\title{
Structural barriers to measles, mumps and rubella (MMR) immunisation uptake in Gypsy, Roma and Traveller communities in the United Kingdom
}

\author{
David Smith ${ }^{\mathrm{a}}$ and Paul Newton ${ }^{\mathrm{b}}$
}

${ }^{a}$ Faculty of Education and Health, University of Greenwich, London, UK; ${ }^{b}$ Faculty of Education and Health, Department of Adult Nursing and Paramedic Science, University of Greenwich, London, UK

\begin{abstract}
Gypsies, Roma and Travellers (GRT) experience a significantly high number of measles cases and have low levels of measles, mumps and rubella (MMR) immunisation. There is little evidence on: why immunisation levels are low; beliefs and practices surrounding the MMR vaccine; or the factors that promote or hinder uptake. This paper presents data from five focus groups with 16 GRT mothers in Kent, South East England. Between them, they had 35 children of whom just under half had not received the course of vaccinations. Focus groups explored the issues GRT parents consider when making vaccination decisions in the context of wider social, ideological, material and practical considerations. Four interrelated themes were identified: way of life and access; engaging with health care staff; perceptions and evaluations of risk; and strategies to minimise MMR-related risks. Our findings provide little support for explanations that emphasise cultural values or practices in shaping immunisation behaviour. Poor service provision, situational constraints related to living circumstances, and multifaceted and severe health issues, which precede and inform decisions over childhood immunisation, were more significant in explaining low uptake.
\end{abstract}

\section{ARTICLE HISTORY}

Received 18 June 2016 Accepted 28 June 2016

\section{KEYWORDS}

MMR; childhood immunisation; Gypsies, Roma and Travellers; ethnicity and health; qualitative study

\section{Introduction}

The resurgence of measles is of considerable public health concern. In recent years, cases have shown a sharp rise, reaching a peak of 6193 in England and Wales in 2013 (Public Health England, 2015). Following a discredited (Taylor et al., 1999) study in 1998 linking the triple measles, mumps and rubella (MMR) vaccine to autism and certain bowel conditions, coverage fell from $92 \%$ in 1995 to $80 \%$ in 2003 . Levels have since risen with $92.3 \%$ of two-year olds having received the MMR vaccination in 2012-2013, which is still below the $95 \%$ required for a population to develop herd immunity (Health \& Social Care Information Centre, 2013). Coverage is also uneven and demonstrates notable geographic and social disparities, with uptake lower among marginalised, economically deprived and/or socially isolated groups. A survey in 25 European countries indicated lower than average MMR coverage in some orthodox religious and anthroposophic groups, economic migrants and Gypsy/Travellers and Roma (GRTs) (O'Flanagan, Cotter, \& Mereckiene, 2010).

The health of GRTs is among the poorest in society with members experiencing a higher burden of illnesses, lower life expectancy and higher infant mortality rates (Cemlyn, Greenfields, Burnett, 
Matthews, \& Whitwell, 2009). Twiselton and Huntington (2009) found that only $20 \%$ of children living in caravans or trailers, and $57 \%$ of those in housing, were fully immunised. Despite a lack of systematic evidence on MMR take-up, one study indicated that only 4 out of 22 Primary Care Trusts (PCTs) estimated coverage of $90 \%$ or above on Gypsy/Traveller sites in their region (Dar, Gobin, Hogarth, Lane, \& Ramsay, 2013). Clusters of measles outbreaks have been reported in GRT communities on several occasions (Health Protection Agency, 2007, 2010). In the Thames Valley region, 63\% of outbreaks between 2006 and 2009 were in GRT communities, which is a 100 times higher incidence than in the general population (Maduma-Butsche \& McCarthy, 2012). Between March and June 2007, 173 cases of measles were reported in seven UK regions of which 156 were in Irish Traveller communities (Cohuet et al., 2009).

Acton, Caffrey, Dunn, and Vinson (1997) argue that the extent to which health inequalities between GRTs and the general population exist could be a statistical artefact. They argue that samples included in GRT health surveys tend to be recruited from poorer members of the GRT community who are known to health visitors and social workers, and that these poorer community members are more likely to experience poorer health. While it is known that GRTs have low levels of MMR coverage, little is known about why uptake is low, the extent to which the organisation and delivery of immunisation services impact on MMR uptake, or how wider social factors shape immunisation decisions.

This paper presents data from a series of focus groups with GRT mothers on their decisions with regard to MMR immunisation and the factors underpinning those decisions. The findings are positioned within a critical realist framework, thus allowing for an exploration of the relative roles of cultural and structural factors in shaping health-related behaviour. Of particular relevance is the proposition that social phenomena are the result of various interacting causalmechanisms: events that occur due to necessity (or the functioning of causal mechanisms) are distinguished from contingent events (occurring due to factors unrelated to those mechanisms) (Dunn, 2012). Disentangling mechanisms that result from deeper social processes and are persistent, cumulative and have tangible outcomes in the social patterning of MMR coverage, from factors that are either extraneous or only tangentially related to the phenomenon in question is the aim of this article. Of further relevance is the proposition that social structures contain certain characteristics (causal powers) that people engage with in the form of stratified material resources (e.g. money, employment or goods) and/or social resources (organisations, social support or information). Individual behaviour is 'reflexive' meaning that people reflect upon and manage their personal concerns in relation to their social context/s, while responding to their social context/s in relation to their personal concerns. Archer (2007) terms these processes as'internal conversations'. This perspective is applied to explore the issues GRT parents consider when making choices about immunisation, contextual issues such as the health care system and the relationship to underlying social structures - which shape people's circumstances and provide the resources through which they experience and understand health and illness. The critical realist approach allowed for the exploration of how the ramifications of the GRT communities' day-to-day socio-material circumstances, shaped their decisions about uptake of the MMR vaccine and their experiences of MMR vaccination services.

\section{Methodology}

A cross-sectional, qualitative study was conducted comprising of 5 focus groups with 16 site-dwelling GRT women $(N=3+3+3+3+4=16)$. Participants were purposively sampled from sites across Kent in South East England and focus groups took place in January 2014. The county was selected as it has the highest population of GRTs in the UK. The 2011 Census counted 5195 Gypsies and Irish Travellers in the county, though analysis of Gypsy and Traveller Accommodation Assessment (GTAA) data indicates

50 a population of 10,931 (Irish Traveller Movement in Britain, 2013). It is likely that the GRT population in Kent (and nationally) is significantly higher: both figures exclude the majority of GRTs who now live in housing, and those who do not disclose their ethnicity. Kent's significant Roma migrant population are also omitted (Smith, 2014). Health workers in Kent have expressed concern at low levels of childhood immunisation among GRT children in the county (Kent \& Medway Public Health Observatory, 2014). 
The focus group guide was developed following a brief open-ended pilot survey about immunisation. The 5 focus groups were held on caravan sites in the locality with a further 16 GRT women and conducted by a trained interviewer from the local travelling community. The women had 35 children between them, of whom 22 were fully or partially immunised with MMR compared to 13 who were not. Participants were from various backgrounds: seven identified as Romany Gypsies, four as Irish Travellers, three as Roma migrants and two as New Travellers. Despite (ethnic and national) diversity in the sample, similarities in terms of a nomadic lifestyle or heritage and distinctive forms of social and economic organisation mean certain important features also unite such groups (Gmelch, 1986). More fundamentally, a common experience of marginalisation, prejudice and exclusion spans these groups and frames their engagement with society, its health systems and immunisation programmes.

Focus groups lasted approximately seventy minutes and data were audio-recorded, transcribed verbatim and analysed for emerging themes before the subsequent focus group. All names used in focus group discussions were changed to pseudonyms after transcription. Transcripts were read by the two authors and a framework of emerging themes were developed by negotiating and agreeing on the content as well as the development of new themes and subthemes (Barbour, 2008). Focus groups were conducted until saturation point was reached. Ethical clearance was gained from the University of Greenwich Research Ethics Committee and procedures regarding informed consent, anonymity and confidentiality were adhered to throughout.

\section{Culture, ethnicity and health}

Ethnicity is frequently invoked in discussions of GRT health and conflated with alleged cultural factors such as fatalism, stoicism, suspiciousness, concepts of hygiene and pollution, attitudes to children and the importance of the oral tradition (Dion, 2008; Vivian \& Dundes, 2004). The concept of ethnicity has attracted critique for entailing essentialist stereotypes and for homogenising people into preconceived categories that minimise differences within specific groups (Campbell \& McLean, 2002). Alleyne (2002) argues that concepts like the 'ethnic community' present an 'epistemological obstacle' to sociologists, as it is frequently used as an explanation rather than something to be explained. When measuring health inequalities, such explanations can complement 'cultural deprivation' frameworks that view the deficient genetic and cultural inheritance of ethno-cultural groups as the primary cause of their poor health (Ahmad \& Bradby, 2007). This perspective also minimises the social processes and mechanisms through which inequalities are generated, and which shape the conditions in which people live.

Nevertheless, ethnicity is an important basis for group membership and collective identity, involving a complex process of classification through which group members differentiate themselves from others (Campbell \& McLean, 2002). When used as an explanatory tool, the generalised and crude depictions of ethnic and/or cultural variables can hinder an understanding of the extent that health inequalities result from ethno-cultural and/or socio-economic factors, the interplay between them or the direction of causal processes. With these caveats in mind, the remainder of the article draws on GRT mothers' accounts of how they assess the risks of non-immunisation in the context of wider social factors and how these in turn influence immunisation decisions. Four interrelated themes were identified: way of life and access; engaging with health care staff; perceptions and evaluations of risk; and strategies to minimise the risks associated with MMR.

\section{The social context of MMR decision-making}

\section{Way of life and health service access}

Health professionals and practitioners often cite cultural barriers as a reason for minority groups' low engagement with health services when questions about the effectiveness of those services are raised (Taylor, 2015). This is particularly true in the case of GRTs where culture is used to explain poor outcomes across the domains of health, education, housing, the criminal justice and state care systems (Smith, 
2016). Scambler (2011) notes that the distribution of health and illness across different population subgroups reveals more about the society that they are located within than its health system. From this perspective, health disparities are closely related to underlying structural and ideological mechanisms, which combine to produce social inequalities and low status. Despite recognition of ethnic and cultural diversity in health service delivery, services have been developed for sedentary populations, resulting in the long-term exclusion of GRTs from services appropriate to their needs (Hester, 2004). Structural barriers and vulnerability to exclusion from health services are therefore a necessary outcome of deeper causal mechanisms, which become embodied and expressed through networks of people in similar structural locations experiencing broadly analogous sets of experiences.

A core theme was the incompatibility of living arrangements and their attendant way of life with the provision of immunisation services. Although the participants were resident on permanent sites, supply side factors such as the location of clinics and health centres meant that attendance was often problematic, due to the isolated location of many sites far from public transport routes.

Most camps are miles away from the shops even now aren't they? It's very rare you'll get one close to shops. Far from doctors, far away from health care, no bus route ... if it's easy access definitely because like I said we don't always live on a bus route. (FG2)

Structural constraints are a greater influence on behaviour in poor and excluded groups, as competing and more immediate concerns take priority over the less immediate benefits of immunisation. In the example below, the participant explains how a combination of strictly demarcated gender roles and short-term material needs can preclude attendance at clinics and surgeries.

If there was a vehicle and the men needed it well you had no vehicle because if they don't go to work we don't eat basically. So if we're left without a vehicle that's the way it is isn't it? (FG2)

Geographic and social isolation also excludes people from the social and material resources necessary for planning a course of action on the basis of the best available knowledge. Conventional methods of health promotion such as information leaflets and letters are less effective at reaching GRT communities, while outreach services are insufficient (Dar et al., 2013). These factors compound the lack of vaccination knowledge and increase reliance on informal sources of information (discussed below).

There are letters and things, I can read a little bit but I still don't understand what they are going on about. (FG1)

A lot of travelling children don't go to school for as long as other children. I don't think they are offered the same information and awareness and what have you. (FG3)

In spite of public health concerns over levels of infectious and communicable diseases in GRT communities, participants argued that outreach services were less frequent than during their own childhoods.

When I was a baby I got school boosters at home, you know into your leg. The health visitor used to come out they came to the camp and gave everybody immunisations. (FG4)

For families who spend extensive periods travelling, the practical obstacles in accessing both immunisation, and health services more generally, are aptly summarised below. The participant explains how increased legal mechanisms to execute speedier evictions of unauthorised sites have adverse health impacts on disrupting health care and the attendant difficulties in accessing vaccination sites.

You get an appointment and then when you get to the appointment you're moved on again. Then if you do get the appointment and you're booked in you'll be moved on again because you never get longer than a week or two weeks is the very most you'll get to stay in one place. (FG3)

These supply side constraints intersect with ideological processes to produce inequitable health outcomes on the one hand, and shared experiences and dispositions towards the health system on the other. More people in the UK admit to feeling ill-disposed towards GRTs (58\%) than any other group (YouGov, 2015) and these wider attitudes can be expressed in the practices of some health staff. Users

50 of public services have long been classified into 'deserving' and 'undeserving' categories through a 'social division of welfare surveillance' that grants significant decision-making autonomy to frontline staff (Henman \& Marston, 2008). It has been reported, for instance, that some general practitioners will register nomadic families as temporary residents (thus excluding them from a range of services) and that others refuse to treat them due to their insistence in having a permanent address (Cemlyn et al., 
2009). Despite these practices constituting indirect and direct discrimination under the Equality Act

2010, participants recalled several occasions when they were denied access.

We're always on the road and you can't get to a clinic or you can't get to a doctor because you have to have a fixed address all the time. (FG5)

I didn't get my child immunised because I travel too much and there wasn't a clinic that would let me immunise him. (FG3)

10 The marginal position and pariah status of GRTs in relation to wider societal institutions is the key to explaining low MMR coverage. These structural determinants not only limit the ability to access and obtain immunisation, but also provide the context within which people respond to health interventions and engage with practitioners on the basis of their own understandings, as outlined below.

\section{Engaging and interacting with health care staff}

Parental rejection of certain vaccinations has been posited as one element of a'symbolic boundary' separating the Gypsy and non-Gypsy world (Feder, Vaclavik, \& Streetly, 1993). Taylor (2015) notes that by locating low uptake in the beliefs and practices of the target population, cultural explanations neglect the possibility of non-compliance representing a strategic opposition to an unwelcomed intrusion into the sphere of family decision-making. Maintaining personal control over their child's health and resisting external pressures to vaccinate was an important strand in some participants' decisions over vaccination.

I refused to give him even though they were all over the place and arguing with me that I'm doing something wrong. (FG3)

I got a letter about the immunisation of my oldest child which is nine years old and never been immunised. They've made an appointment, if I don't want her to have it I'll have to cancel it. I said 'I didn't ask you to make an appointment.' (FG1)

Poor relations between GRTs and health staff contribute to low take up of preventative programmes (Parry et al., 2004; Smith \& Ruston, 2013). When mistrust is emphasised as a cause of non-compliance, it is generally viewed as part of an antagonistic world view towards society and the medical profession. Sociological perspectives argue that lived reality shapes behaviour to a larger extent than shared cultural values or world views. Many GRTs report experiencing discrimination and exclusion on a regular basis with suspicion and apprehension towards societal institutions and their representatives often consistent with situational factors, rather than evidence of a distinctive value system. Further, such sentiments are not exceptional and mirror increasing public scepticism towards official health advice and biomedical treatment and prevention of disease (Broom \& Tovey, 2008).

They don't tell you the side effects of having these vaccines they don't tell you this side of it. They only tell you that yes it will stop measles, mumps and rubella they won't tell you that it causes your child to have fits later on in life. (FG5)

Parry et al. (2004) identified how the anticipation of conflict with health staff generated a defensively hostile' demeanour among many GRTs and an aversion to using health services.

The thing is I trust nature more than I trust professionals to be honest. Unless I know that professional personally then I would trust mother-nature because I know her personally. (FG3)

Negative experiences in health care settings and a perception that their health is afforded a low priority confirmed through the paucity of engagement with this community, played a significant role in some mothers' refusal to engage with vaccination programmes.

They [health professionals] don't care they don't really care about this population. If they would care about this population they will make the effort to pop along. (FG2)

\section{Evaluating and minimising risk}

GRTs evaluate health risks through emphasising risks generated through assimilatory policies of spatial control, poor living conditions, limited access to health care and their own behaviour (Ruston \& Smith, 2013). Due to the high incidence of measles in GRT communities, most participants had personal 
experience of the disease, either in their own or other families, and had a clear understanding of how such diseases are transmitted. They provided several examples of measles being spread among children, generally following attendance at social events (Cohuet et al., 2009).

The kids were in hospital with measles, remember? That was around your Mol's funeral, that's where they picked it up you know my Bobby picked it up at Mol's funeral. (FG1)

Everyone you spoke to then, someone had it. It was like wildfire wasn't it going through the travellers. It spread so fast. (FG1)

Cultural propensities including an overly permissive attitude to child rearing have been identified as a further contributor to the poor health experienced by GRT children and throughout the life cycle (Dion, 2008). Focus group narratives indicate an opposite chain of causation: rather than a culturally shaped indulgence towards children leading to their ill health, it is high levels of childhood illness that influence parental attitudes towards their children whom adults regard as being inherently vulnerable and prone to future illnesses. These attitudes and perceptions are contingent however and do not act in a uniform direction as cultural accounts suggest, but act in more subtle and divergent ways to produce multiple outcomes. The perception of their children's health as precarious made some mothers extremely averse to causing them unnecessary pain and suffering.

Nothing could encourage me to have a needle put in my baby's arm or leg and scream his fucking head off. (FG3) Others considered that frequent bouts of ill health made their children more prone to contracting infectious illnesses. This influenced some participants to immunise their children with MMR, to prevent further illness and bolster weakened immune systems.

I'm definitely going to get him [son] done with the MMR because he's been so sick since he's born anyway with viruses and ear infections. (FG 4)

However, the inverse was also apparent. Recurrent and/or serious childhood illnesses caused some mothers to fear that their children were predisposed to harmful effects from the MMR vaccine, making them reluctant to vaccinate. Others argued that potential side effects were worse than the diseases it was produced to prevent.

... When he was small - he was really small, he got pneumonia. Because he'd had pneumonia I didn't want to give him the needle either. (FG2)

Allergic reactions are worse than the measles. My daughter could have died. Measles can be treated when caught. (FG5)

The decision whether or not to immunise is also informed by personal concerns and experiences. For one mother, first-hand experience of her son suffering from measles was sufficient impetus for her to have him vaccinated later.

I thought my child was going to die. Honest to God. As soon as them measles were gone I marched him straight down and got the MMR.(FG1)

The experiences of other family and community members, whose children had previously received $M M R$, could shape behaviour in both directions however. One participant commented that:

My sister gave it to all her children and they were OK so I'm giving it to mine. (FG4)

\section{Timing and risk}

40 Timing of the injections played a pivotal role in influencing decision-making and immunisation behaviour in two ways: first in terms of the age it is administered, and secondly in a general opposition to the combined vaccination. Many considered the recommended age of 12-13 months for the first MMR dose too young, and there was a consensus that it should be given when children are older and their immune systems stronger to minimise the risks associated with MMR. In many cases, non-immunisation represents a decision to postpone, not reject, the MMR vaccine.

My GP has sent me letters about MMR but I will when he is old enough. (FG4)

Travellers don't get it now until your children go over five, they won't get it. They reckon to get it later on. (FG2) 
There was also a clear preference for spacing immunisations individually, since receiving three vaccines simultaneously was considered excessive for young children. Separate injections are not available on the NHS and the costs of receiving them privately meant this was not an option for many.

It's a really unnecessary overload into such a young body if you just dump so much into it. This is why so many children get ill. (FG3)

I think they put a price on it if you want it individually and I couldn't do that. (FG5)

Relatively low levels of engagement with health services and the paucity of targeted information meant that family and friends are often the main source of health information and certain beliefs - such as those concerning the safest time and method of receiving MMR - are commonly held (Smith \& Ruston, 2013). The structure of social relations in GRT communities also means that gossip and anecdotes circulate widely, and constitute a further dimension of decision-making.

Jen who has the autistic child she believes it's that [MMR] so that's why people will hold back because of that especially everybody who knows her and her child. (FG4)

Just visiting other travellers, then if someone tells family they think something's going to happen to their baby, they're not going to take a risk. (FG2)

Few participants were opposed to MMR in principle and objections were likely to be practical or pragmatic in nature. As studies of other under-immunised communities have indicated (Henderson, Millett, \& Thorogood, 2008), there was no consensus for or against the vaccine and no cohesive rationale underpinning immunisation decisions. Decisions whether or not to immunise are not monolithic therefore and reflected the dynamic intersection of structural forces, normative influences and individual circumstances, which shape decision-making in contradictory directions. There was no apparent cultural orientation towards childhood immunisation; rather a generalised wariness of health professionals and interventions along with elements of shared beliefs about when and how MMR should be provided, which combined to frame participants' decisions.

\section{Policy implications}

The notion that GRTs are inalienably 'different' and 'hard to reach' has legitimised the minimal progress in reducing inequalities experienced by members of these communities. The challenges for service provision include similarities with other marginalised groups, though reducing inequalities in MMR uptake needs to be particularly concentrated due to the more intense disadvantages that members of GRT communities face (Carr et al., 2014). Masseria, Mladovsky, and Hernandez-Quevedo (2010) note that structural factors such as low levels of education, poverty and exclusion are the main determinants of Roma health inequalities and recommend policies oriented towards reducing poverty, raising educational outcomes and increasing social integration to mitigate health inequalities. Variability in uptake of MMR raises the potential of promoting the benefits of immunisation via community networks (Henderson et al., 2008). Outreach programmes have been identified as an effective engagement strategy, the success of which depends on the nature of interpersonal relations between the outreach worker and community. Trust, social influence and the ability to negotiate the focus of the intervention are the crucial variables in ensuring the success or otherwise of health interventions targeted at this group (Lhussier, Carr, \& Forster, 2016). Following an outbreak of measles on a site in Manchester, a key factor containing its spread was daily visits by the same health visitor. This allowed for a working relationship based on mutual trust to develop between the health visitor and site residents, who could voice their concerns over MMR and readily obtain health advice. Of the 56 eligible children, 55 were vaccinated with MMR either at the site or later at a local general practice surgery, and only 1 secondary case of measles occurred on the site (Reynolds et al., 2008). Findings indicate that health beliefs are not the major factor in explaining low MMR uptake in GRT communities. Of greater significance are the organisation and delivery of immunisation services and the effects of long-standing, pre-existing health inequalities which may exacerbate existing barriers to immunisation. Findings suggest uptake could be increased through the use of community outreach workers with the features identified by Lhussier 
et al. (2016). These could promote a more flexible approach to MMR immunisation that includes the option of separate injections and immunisation on sites, increasing the uptake of the MMR vaccination in GRT communities.

\section{Conclusion}

Arguments over the relative influence of cultural versus structural factors in producing health inequalities have important implications for engaging marginalised populations in preventative programmes and hinge on opposite views of causation. In the first view, cultural beliefs and practices are responsible for generating social outcomes, while in the second, social context is responsible for shared behavioural and experiential patterns. Accounts emphasising the causative role of culture in shaping willingness to access immunisation, or health services more generally, tend to employ circular reasoning, fail to clearly specify cultural variables and are tautologies where values are inferred from behaviour, and the behaviour explained with reference to a purported value system. By contrast, much more significant factors in explaining low MMR uptake are the lack of tailored services; situational constraints based in historical and contemporary experiences of marginalisation; and the existence of multifaceted and severe social and health inequalities, which precede and inform decisions surrounding vaccination. Unlike previous studies which attach a significant causal role to cultural values and world views (Honer \& Hoppie, 2004; Van Cleemput, Parry, Thomas, Peters, \& Cooper, 2007), our findings indicate that collective perceptions and orientations towards MMR represent adaptive and strategic responses to deeper structural factors that manifest themselves in high levels of unemployment, economic inactivity, poverty, poor living conditions and both social and spatial exclusion. GRT parents have a clear understanding of the risks of immunisation and non-immunisation, and do not hold beliefs that are drastically different to those found in the wider population (Poltorak, Leach, Fairhead, \& Cassell, 2005). What is drastically different is the context in which decisions are made, as parents mediate between their structural circumstances and personal health beliefs, which are largely shaped by those circumstances and the effects of poor health status.

\section{Disclosure statement}

No potential conflict of interest was reported by the authors.

\section{Funding}

This work was supported by the RAE Competitive Investment Round Research and Enterprise Investment Programme 2013/14.

\section{References}

Acton, T., Caffrey, S., Dunn, S., \& Vinson, P. (1997). Gender issues in accounts of Gypsy health and hygiene as discourses of social control. InT. Acton \& G. Munday (Eds.), Romani culture and Gypsy identity. Hatfield: University of Hertfordshire Press.

Ahmad, W., \& Bradby, H. (2007). Locating ethnicity and health: Exploring concepts and contexts. Sociology of Health \& Illness, 29, 795-810.

Alleyne, B. (2002). An idea of community and its discontents: Towards a more reflexive sense of belonging in multicultural Britain. Ethnic and Racial Studies, 25, 607-627.

Archer, M. S. (2007). Making our way through the world: Human reflexivity and social mobility. Cambridge: Cambridge University Press.

Barbour, R. (2008). Doing focus groups. London: Sage.

Broom, A., \& Tovey, P. (2008). Therapeutic pluralism: Exploring the experiences of cancer patients and professionals. London: Routledge.

Campbell, C., \& McLean, C. (2002). Representations of ethnicity in people's accounts of local community participation in a multi-ethnic community in England. Journal of Community and Applied Social Psychology, 12, 13-29.

Carr, S., Lhussier, M., Forster, N., Goodall, D., Geddes, L., Pennington, M., ... Michie, S. (2014). Outreach programmes for health improvement of Traveller communities: A synthesis of evidence. Public Health Research, 2. 
Cemlyn, S., Greenfields, M., Burnett, S., Matthews, Z., \& Whitwell, C. (2009). Inequalities experienced by Gypsy and Traveller communities: A review. London: Equalities and Human Rights Commission.

Cohuet, S., Bukasa, A., Heathcock, R., White, J., Brown, K., Ramsay, M., \& Fraser, G. (2009). A measles outbreak among the Irish travellers ethnic group after a funeral. England, March to June 2007. Epidemiology \& Infection, 137, 1759-1765. doi: $10.1017 /$ S0950268809002714

Dar, O., Gobin, M., Hogarth, S., Lane, C., \& Ramsay, M. (2013). Mapping the Gypsy Traveller community in England:What we know about their health service provision and childhood immunization uptake. Journal of Public Health, 35, 404-412.

Dion, X. (2008). Gypsies and Travellers: Cultural influences on health. Community Practitioner, 81, 31-34.

Dunn, J. R. (2012). Explanation, philosophy and theory in health inequalities research. In P. O'Campo \& J. R. Dunn (Eds.), Rethinking social epidemiology. London: Springer.

Feder, G., Vaclavik, T., \& Streetly, A. (1993). Traveller Gypsies and childhood immunization: A study in east London. British Journal of General Practice, 43, 281-284.

Gmelch, S. B. (1986). Groups that don't want in: Gypsies and other artisan, trader and entertainment minorities. Annual Review of Anthropology, 15, 307-330.

Health Protection Agency. (2007). Health protection report (Vol. 1, No. 21). Retrieved from http://webarchive.nationalarchives. gov.uk/20140714084352/http://www.hpa.org.uk/hpr/archives/2007/hpr2107.pdf

Health Protection Agency. (2010). Health protection report (Vol. 4, No. 30). Retrieved from http://webarchive.nationalarchives. gov.uk/20140714084352/http://www.hpa.org.uk/hpr/archives/2010/hpr3010.pdf

Health and Social Care Information Centre. (2013). NHS immunisation statistics 2012-13. Author.

Henderson, L., Millett, C., \& Thorogood, N. (2008). Perceptions of childhood immunization in a minority community: Qualitative study. Journal of the Royal Society of Medicine, 101, 244-251.

Henman, P., \& Marston, G. (2008). The social division of welfare surveillance. Journal of Social Policy, 37, 186-205.

Hester, R. (2004). Services provided to Gypsy/Traveller children: A review of the current literature for the National Evaluation of the Children's Fund. Birmingham: NECF.

Honer, D., \& Hoppie, P. (2004). The enigma of the Gypsy patient. Modern Medicine Network. Retrieved from http://www. modernmedicine.com/modern-medicine/content/enigma-gypsy-patient

Irish Traveller Movement in Britain. (2013). Gypsy and Traveller population in England and the 2011 Census. London: ITMB.

Kent and Medway Public Health Observatory. (2014). JSNA Gypsy/Travellerpopulations 2013/14. Retrieved from http://www. kmpho.nhs.uk/search/?q=gypsies

Lhussier, M., Carr, S. M., \& Forster, N. (2016). A realist synthesis of the evidence on outreach programmes for health improvement of Traveller Communities. Journal of Public Health, 38, e125-e132.

Maduma-Butsche, A., \& McCarthy, N. (2012). The burden and impact of measles among the Gypsy-Traveller communities, Thames Valley, 2006-2009. Journal of Public Health, 35, 27-31.

Masseria, C., Mladovsky, P., \& Hernandez-Quevedo, C. (2010). The socio-economic determinants of the health status of Roma in comparison with non-Roma in Bulgaria, Hungary and Romania. The European Journal of Public Health, 20, $549-554$.

O'Flanagan, D., Cotter, S., \& Mereckiene, J. (2010). Analysis of determinants for low MMR vaccination coverage in Europe, 2010. Venice II Consortium, WHO.

Parry, G., Van Cleemput, P., Peters, J., Moore, J., Walters, S., Thomas,K., \& Cooper, C. (2004) The health status of Gypsies and Travellers in England (Report of Dept of Health Inequalities in Heath Research Initiative Project 121/7500). University of Sheffield.

Poltorak, M., Leach, M., Fairhead, J., \& Cassell, J. (2005). 'MMR talk' and vaccination choices: An ethnographic study in Brighton. Social Science and Medicine, 61, 709-719.

Public Health England. (2015). Measles notifications in England and Wales 2011-2014. Retrieved from https://www.gov. uk/government/publications/measles-notifications-by-age-group-region-and-sex/measles-notifications-in-englandand-wales-by-region

Reynolds, F., Petroyic, M., Will, S., Dutton, A., Paver, K., Kirkpatrick, A., \& Kempster, J. (2008). Management of measles in a traveller community: Public health issues of trust, choice and communication. Public Health, 122, 390-393.

Ruston, A. M., \& Smith, D. (2013). Gypsies/Travellers and health: Risk categorisation versus being 'at risk'. Health, Risk and Society, 15, 176-193.

Scambler, G. (2011). 'Tackling health inequalities' and its pros, cons and contradictions: A commentary on Blackman, Wistow and Byrne. Social Science \& Medicine, 72, 1975-1977.

Smith, D. (2014). Local engagement Roma integration project: UK feasibility report. Fundamental Rights Agency.

Smith, D. (2016). The political context of migration in the UK: The case of Roma Gypsies. In N. Zakaria (Ed.), Handbook of research on the impacts of international business and political affairs on the global economy. IGI Global.

Smith, D., \& Ruston, A. M. (2013). 'If you feel that nobody wants you you'll withdraw into your own': Gypsies/Travellers, networks and healthcare utilisation. Sociology of Health and IIIness, 35, 1196-1210.

Taylor, A. J. (2015). Culture and behaviour in mass health interventions: Lessons from the global polio eradication initiative. Critical Public Health, 25, 192-204.

Taylor, B., Miller, E., Farrington, C. P., Petropoulos, M. C., Favot-Mayaud, I., Li, J., \& Waight, P. A. (1999). Autism and measles, mumps, and rubella vaccine: No epidemiological evidence for a causal association. The Lancet, 353, $2026-2029$.

Twiselton, I., \& Huntington, F. (2009). Health needs assessment: Cumbria Gypsies Travellers. NHS Cumbria. 
Van Cleemput, P., Parry, G., Thomas, K., Peters, J., \& Cooper, C. (2007). Health related beliefs and experiences of Gypsies and Travellers: A qualitative study. Journal of Epidemiology and Community Health, 61, 205-210.

Vivian, C., \& Dundes, L. (2004). The crossroads of culture and health among the Roma (Gypsies). Journal of Nursing Scholarship, 36, 86-91.

YouGov. (2015). Roma people and Muslims are the least tolerated minorities in Europe. Retrieved from https://yougov. co.uk/news/2015/06/05/european-attitudes-minorities/

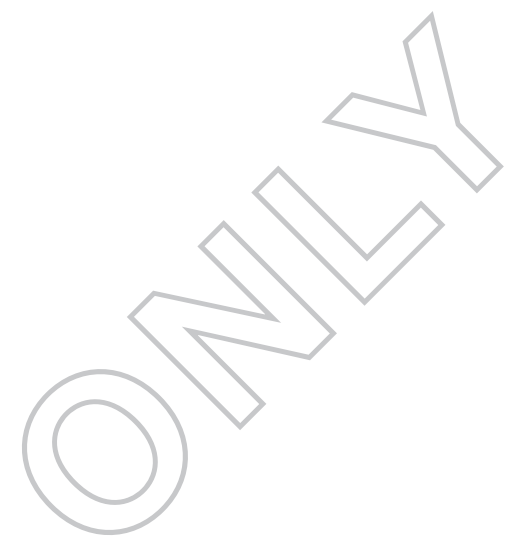

